\title{
Development of Multi-Polarity Arithmetic-Haar Transform and Its Applications in Logic Design
}

\author{
Bogdan J. Falkowski and Shixing Yan \\ School of Electrical and Electronic Engineering, Nanyang Technological University \\ Block S1, 50 Nanyang Avenue, Singapore 639798
}

\begin{abstract}
The generalization of multi-polarity arithmeticHaar transform in the form of layered Kronecker matrices is proposed in this article. The new recursive relations are given in the form of layered Kronecker matrices and hence they have fast transforms and are computationally advantageous.
\end{abstract}

\section{INTRODUCTION}

Both the Haar wavelet transform (non-normalized version of the transform where only signs are entered into the transform matrix) and arithmetic transform have been used in many applications of logic design [1]-[3]. As each of these transforms has same advantages and disadvantages, it is also beneficial to calculate the spectrum of a logic function by means of some other known spectrum of the same function without needing to regain the original function. Such a conversion for arithmetic and Haar spectra for arbitrary $n$ were shown in [4]. In [5] an idea of a combined arithmetic-Haar transform was proposed. Such a transform was defined for the first eight functions and experimental results shown in [5] proved that arithmetic-Haar transform is more efficient than other used transforms in logic design such as Walsh, Haar and arithmetic for some benchmark functions. Therefore it is interesting not only theoretically but also practically, to extend this arithmeticHaar transform for higher matrix dimensions. In our previous article [6], we showed arithmetic-Haar transform for arbitrary matrices in zero polarity, while in this article for the first time the multi-polarity arithmetic-Haar transform is introduced and this is our main contribution. In addition, we propose the generalization of multi-polarity arithmetic-Haar transform with fast algorithms.

\section{BASIC DEFINITIONS}

Logical expansions are useful to represent logic functions, which usually use AND and EXOR $(\oplus)$ operators. There are types of expansion including Shannon expansion [2], [7] and Davio expansion [2], [7] which are widely used in logic design . For an $n$-variable function $f\left(x_{1}, x_{2}, \cdots, x_{i}, \cdots, x_{n-1}, x_{n}\right)$, $f_{\mid x_{i}=b}=f\left(x_{1}, x_{2}, \cdots, x_{i-1}, b, x_{i+1}, \cdots, x_{n-1}, x_{n}\right)$ where $1 \leq i \leq n$ and $b=0$ or 1 .

Definition 1: Shannon expansion for the $n$-variable function can be represented as:

$$
f=x_{i} f_{\mid x_{i}=1} \oplus \bar{x}_{i} f_{\mid x_{i}=0} .
$$

Definition 2: There are two types of Davio expansions, including positive Davio expansion and negative Davio expansion. For the $n$-variable function, the positive and negative Davio expansions are represented by (2) and (3), respectively.

$$
\begin{aligned}
& f=f_{\mid x_{i}=0} \oplus x_{i}\left(f_{\mid x_{i}=0} \oplus f_{\mid x_{i}=1}\right) \\
& f=f_{\mid x_{i}=1} \oplus \bar{x}_{i}\left(f_{\mid x_{i}=0} \oplus f_{\mid x_{i}=1}\right)
\end{aligned}
$$

Let the symbol ' $\otimes$ ' represent Kronecker product [2], [7] of two matrices.

Definition 3: Let the symbol $\vec{\alpha}_{n}=\left\{\alpha_{1}, \alpha_{2}, \cdots, \alpha_{i}, \cdots\right.$, $\left.\alpha_{n-1}, \alpha_{n}\right\}$ represent the polarity of the multi-polarity Walsh transform. The forward multi-polarity Walsh transform matrix of order $2^{n}$ is defined as [8]:

$$
M W^{\vec{\alpha}_{n}}(n)=\otimes_{i=1}^{n} M W^{\alpha_{i}}(1)
$$

where $M W^{\alpha_{i}}(1)=\left\{\begin{array}{rr}{\left[\begin{array}{rr}1 & 1 \\ 1 & -1\end{array}\right] \quad \text { if } \quad \alpha_{i}=0,} \\ 1 & 1 \\ -1 & 1\end{array}\right] \quad$ if $\quad \alpha_{i}=1$.

Definition 4: Let the symbol $\vec{\beta}_{n}=\left\{\beta_{1}, \beta_{2}, \cdots, \beta_{i}, \cdots\right.$, $\left.\beta_{n-1}, \beta_{n}\right\}$ represent the polarity of the multi-polarity arithmetic transform. The forward multi-polarity arithmetic transform matrix of order $2^{n}$ is defined as [9]:

$$
M A^{\vec{\beta}_{n}}(n)=\otimes_{i=1}^{n} M A^{\beta_{i}}(1)
$$

where $M A^{\beta_{i}}(1)=\left\{\begin{array}{rr}{\left[\begin{array}{rr}1 & 0 \\ -1 & 1\end{array}\right] \quad \text { if } \beta_{i}=0,} \\ 0 & 1 \\ 1 & -1\end{array}\right] \quad$ if $\beta_{i}=1$.

Definition 5: Let the symbol $\vec{\gamma}_{n}=\left\{\gamma_{1}, \gamma_{2}, \cdots, \gamma_{i}, \cdots\right.$, $\left.\gamma_{n-1}, \gamma_{n}\right\}$ represent the polarity of the multi-polarity Haar transform. The forward multi-polarity Haar transform matrix 
of order $2^{n}$ is defined as [10]:

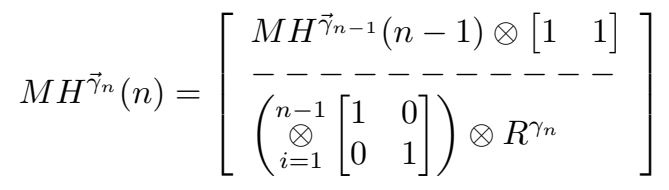

$$
\begin{aligned}
& \text { where } M H^{\gamma_{i}}(1)=\left\{\begin{array}{rr}
{\left[\begin{array}{rr}
1 & 1 \\
1 & -1
\end{array}\right] \quad \text { if } \quad \gamma_{i}=0} \\
1 & 1 \\
-1 & 1
\end{array}\right] \quad \text { if } \quad \gamma_{i}=1 \\
& \text { and } R^{\gamma_{i}}(1)= \begin{cases}{\left[\begin{array}{ll}
1 & -1 \\
-1 & 1
\end{array}\right] \quad \text { if } \quad \gamma_{i}=0} \\
\text { if } & \gamma_{i}=1\end{cases}
\end{aligned}
$$

\section{Multi-Polarity ARITHMEtiC-HaAR Transform}

Let $\vec{\omega}_{n}=\left\{\omega_{1}, \omega_{2}, \cdots, \omega_{i}, \cdots, \omega_{n-1}, \omega_{n}\right\}$ be $n$-tuple over $\mathrm{GF}(2)$. This vector represents the polarity of multi-polarity arithmetic-Haar transform, $n, i$ are integers and $1 \leq i \leq n$. For an $n$-variable function $f\left(x_{1}, x_{2}, \cdots x_{r}, \cdots x_{n-1}, x_{n}\right)$, the basic functions for the multi-polarity arithmetic-Haar expansions can be combined from two sets of basic functions. The first $2^{n-1}$ basic functions are generated from the positive Davio expansion for variables $\dot{x}_{1}$ to $\dot{x}_{n}$, excluding $\dot{x}_{r}$ where $1 \leq r \leq n$ :

$$
\begin{array}{r}
{\left[\begin{array}{ll}
1 & \dot{x}_{1}
\end{array}\right] \otimes\left[\begin{array}{ll}
1 & \dot{x}_{2}
\end{array}\right] \otimes \cdots \otimes\left[\begin{array}{ll}
1 & \dot{x}_{r-1}
\end{array}\right] \otimes\left[\begin{array}{ll}
1 & \dot{x}_{r+1}
\end{array}\right] \otimes} \\
\cdots \otimes\left[\begin{array}{ll}
1 & \dot{x}_{n-1}
\end{array}\right] \otimes\left[\begin{array}{ll}
1 & \dot{x}_{n}
\end{array}\right] .
\end{array}
$$

For the other $2^{n-1}$ basic functions, the functions can be generated from multiplying the Shannon expansion for variables $x_{1}$ to $x_{n}$, excluding $x_{r}$ :

$$
\begin{aligned}
& {\left[\begin{array}{ll}
\bar{x}_{1} & x_{1}
\end{array}\right] \otimes\left[\bar{x}_{2} \quad x_{2}\right] \otimes \cdots \otimes\left[\bar{x}_{r-1} \quad x_{r-1}\right] \otimes} \\
& {\left[\begin{array}{ll}
\bar{x}_{r+1} & x_{r+1}
\end{array}\right] \otimes \cdots \otimes\left[\bar{x}_{n-1} \quad x_{n-1}\right] \otimes\left[\begin{array}{ll}
\bar{x}_{n} & x_{n}
\end{array}\right]} \\
& \text { by }\left(1-2 \dot{x}_{r}\right) \text {. }
\end{aligned}
$$

In (7) and (8), $\dot{x}_{i}=\left\{\begin{array}{ll}x_{i}, & \text { if } \omega_{i}=0, \\ \bar{x}_{i}, & \text { if } \omega_{i}=1,\end{array}\right.$ where $1 \leq i \leq n$.

Such an approach allows us to generalize the multi-polarity arithmetic-Haar matrices and corresponding expansions in many ways by selecting the polarity $\vec{\omega}_{n}$ and the order $r$, so this is a general method that can provide compact spectral representation with many zeros for any $n$-variable logic function.

Definition 6: From the multi-polarity arithmetic-Haar expansions, the $r$ th-order multi-polarity arithmetic-Haar transform matrix $A H_{r}(n)$ and its inverse $A H_{r}^{-1}(n)$ can be defined as:

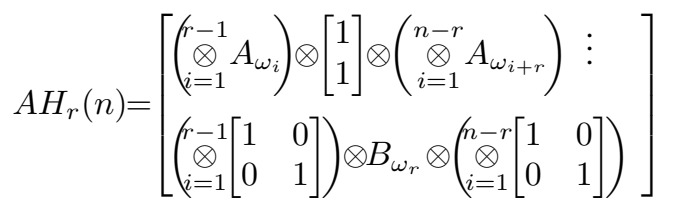

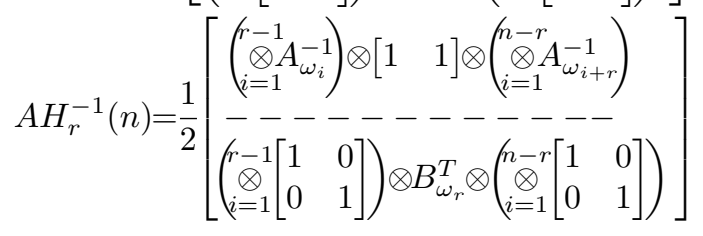

$$
\begin{aligned}
& A_{\omega_{k}}=\left\{\begin{array}{ll}
{\left[\begin{array}{ll}
1 & 0 \\
1 & 1
\end{array}\right] \quad \text { if } \omega_{k}=0,} \\
{\left[\begin{array}{ll}
1 & 1 \\
1 & 0
\end{array}\right] \quad \text { if } \omega_{k}=1,}
\end{array} \quad A_{\omega_{k}}^{-1}= \begin{cases}{\left[\begin{array}{rr}
1 & 0 \\
-1 & 1
\end{array}\right] \quad \text { if } \omega_{k}=0,} \\
{\left[\begin{array}{rr}
0 & 1 \\
1 & -1
\end{array}\right] \quad \text { if } \omega_{k}=1,}\end{cases} \right. \\
& B_{\omega_{k}}=\left\{\begin{array}{l}
{\left[\begin{array}{r}
1 \\
-1
\end{array}\right] \quad \text { if } \omega_{k}=0,} \\
{\left[\begin{array}{r}
-1 \\
1
\end{array}\right] \quad \text { if } \omega_{k}=1,}
\end{array} \text { and } B_{\omega_{k}}^{T}= \begin{cases}{\left[\begin{array}{ll}
1 & -1
\end{array}\right]} & \text { if } \omega_{k}=0, \\
{\left[\begin{array}{ll}
-1 & 1
\end{array}\right]} & \text { if } \omega_{k}=1,\end{cases} \right.
\end{aligned}
$$

where $n=2,3,4 \cdots, 1 \leq r \leq n$ and $1 \leq k \leq n$.

In the above equations, the symbol $\otimes_{i=1}^{j}$, represents the Kronecker product of $j$ matrices. When the Kronecker product of $j$ matrices is carried out for the above equations for $j=0$, then the term ${ }_{i=1}^{j}$, disappears from the above equations. The vertical dotted lines denote the layered vertical Kronecker matrices, and the horizontal dashed lines denote the layered horizontal Kronecker matrices, respectively. The layered horizontal Kronecker matrix is defined as the horizontal sum of Kronecker matrices, and the layered vertical Kronecker matrix is defined as the vertical sum of Kronecker matrices [4].

Example 1: For $n=3, r=2$ and $\vec{\omega}_{3}=\left\{\omega_{1}, \omega_{2}, \omega_{3}\right\}=$ $\{0,1,1\}$, the arithmetic-Haar forward transform matrix $\mathrm{AH}_{2}(3)$ is:

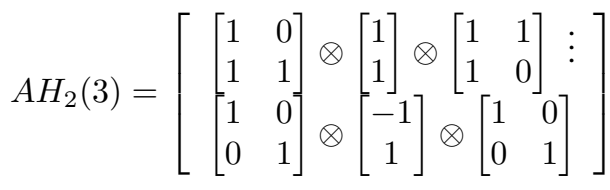

$$
\begin{aligned}
& =\left[\begin{array}{rrrrrrrr}
1 & 1 & 0 & 0 & -1 & 0 & 0 & 0 \\
1 & 0 & 0 & 0 & 0 & -1 & 0 & 0 \\
1 & 1 & 0 & 0 & 1 & 0 & 0 & 0 \\
1 & 0 & 0 & 0 & 0 & 1 & 0 & 0 \\
1 & 1 & 1 & 1 & 0 & 0 & -1 & 0 \\
1 & 0 & 1 & 0 & 0 & 0 & 0 & -1 \\
1 & 1 & 1 & 1 & 0 & 0 & 1 & 0 \\
1 & 0 & 1 & 0 & 0 & 0 & 0 & 1
\end{array}\right],
\end{aligned}
$$


and its inverse $A H_{2}^{-1}(3)$ is:

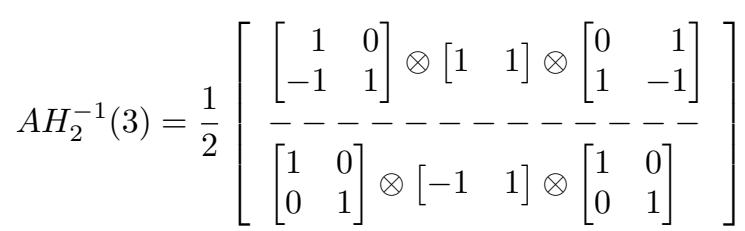

$$
\begin{aligned}
& =\frac{1}{2}\left[\begin{array}{rrrrrrrr}
0 & 1 & 0 & 1 & 0 & 0 & 0 & 0 \\
1 & -1 & 1 & -1 & 0 & 0 & 0 & 0 \\
0 & -1 & 0 & -1 & 0 & 1 & 0 & 1 \\
-1 & 1 & -1 & 1 & 1 & -1 & 1 & -1 \\
-1 & 0 & 1 & 0 & 0 & 0 & 0 & 0 \\
0 & -1 & 0 & 1 & 0 & 0 & 0 & 0 \\
0 & 0 & 0 & 0 & -1 & 0 & 1 & 0 \\
0 & 0 & 0 & 0 & 0 & -1 & 0 & 1
\end{array}\right] \text {. }
\end{aligned}
$$

Comparatively, the most known transform which has been applied to many areas including logic synthesis and optimization is Walsh transform. Similarly, the proposed multipolarity arithmetic-Haar transform can also be used for the logic design. The following example shows the applications of Walsh and arithmetic-Haar transforms to the same function.

Example 2: Consider a 3-variable function $f_{1}\left(x_{1}, x_{2}, x_{3}\right)$ $=\sum(0,2,4,5,7)$. Using the multi-polarity arithmetic-Haar transform in Example 1 and corresponding 8-point Walsh transform for $f_{1}$, the corresponding arithmetic-Haar coefficients and Walsh coefficients can be calculated respectively as follow:

$$
\left.\left.A H_{2}^{-1}(3) \times\left[\begin{array}{l}
1 \\
0 \\
1 \\
0 \\
1 \\
1 \\
0 \\
1
\end{array}\right]=\frac{1}{2}\left[\begin{array}{r}
0 \\
2 \\
2 \\
-3 \\
0 \\
0 \\
-1 \\
0
\end{array}\right],\left(\begin{array}{c}
3 \\
\otimes \\
i=1
\end{array}\right] \begin{array}{cc}
1 & 1 \\
1 & -1
\end{array}\right]\right)^{-1} \times\left[\begin{array}{l}
1 \\
0 \\
1 \\
0 \\
1 \\
1 \\
0 \\
1
\end{array}\right]=\frac{1}{8}\left[\begin{array}{r}
5 \\
1 \\
1 \\
1 \\
-1 \\
3 \\
-1 \\
-1
\end{array}\right] .
$$

Using (7) and (8) the corresponding arithmetic-Haar expansion for the function $f_{1}$ is:

$$
f_{1}=\frac{1}{2}\left[2 \bar{x}_{3}+2 x_{1}-3 x_{1} \bar{x}_{3}-x_{1} \bar{x}_{3}\left(1-2 \bar{x}_{2}\right)\right] .
$$

From the Walsh coefficients calculated above, the corresponding Walsh polynomial expansion for the function $f_{1}$ is:

$$
\begin{array}{r}
f_{1}=\frac{1}{8}\left[5+\left(1-2 x_{3}\right)+\left(1-2 x_{2}\right)+\left(1-2 x_{2}\right)\left(1-2 x_{3}\right)\right. \\
-\left(1-2 x_{1}\right)+3\left(1-2 x_{1}\right)\left(1-2 x_{3}\right)-\left(1-2 x_{1}\right)\left(1-2 x_{2}\right) \\
-\left(1-2 x_{1}\right)\left(1-2 x_{2}\right)\left(1-2 x_{3}\right) .
\end{array}
$$

It is clearly shown that the arithmetic-Haar expansion is much shorter than the corresponding Walsh expansion for this function.

Since the multi-polarity arithmetic-Haar transform has the recursive relations given in the form of layered Kronecker matrices, it is possible to derive fast algorithm for the calculation of the multi-polarity arithmetic-Haar transform matrices. Similar to the fast Walsh transform and other known fast transforms, the multi-polarity arithmetic-Haar transform matrix can be calculated by the products of the factored matrices. The fast algorithms give us a way to calculate the transform more efficiently. Furthermore, the butterfly diagrams can be developed based on the factorized matrices, which can be used for the hardware implementation of the transform.

Example 3: The multi-polarity arithmetic-Haar transform $\mathrm{AH}_{2}(3)$ and $\mathrm{AH}_{2}^{-1}(3)$ in Example 1 can be factorized to the products of three matrices by fast algorithms as follows:

$$
\begin{aligned}
A H_{2}(3)= & {\left[\begin{array}{rrrrrrrr}
1 & 0 & -1 & 0 & 0 & 0 & 0 & 0 \\
0 & 1 & 0 & -1 & 0 & 0 & 0 & 0 \\
1 & 0 & 1 & 0 & 0 & 0 & 0 & 0 \\
0 & 1 & 0 & 1 & 0 & 0 & 0 & 0 \\
0 & 0 & 0 & 0 & 1 & 0 & -1 & 0 \\
0 & 0 & 0 & 0 & 0 & 1 & 0 & -1 \\
0 & 0 & 0 & 0 & 1 & 0 & 1 & 0 \\
0 & 0 & 0 & 0 & 0 & 1 & 0 & 1
\end{array}\right] \times } \\
& {\left[\begin{array}{llllllll}
1 & 0 & 0 & 0 & 0 & 0 & 0 & 0 \\
0 & 1 & 0 & 0 & 0 & 0 & 0 & 0 \\
0 & 0 & 0 & 0 & 1 & 0 & 0 & 0 \\
0 & 0 & 0 & 0 & 0 & 1 & 0 & 0 \\
1 & 0 & 1 & 0 & 0 & 0 & 0 & 0 \\
0 & 1 & 0 & 1 & 0 & 0 & 0 & 0 \\
0 & 0 & 0 & 0 & 0 & 0 & 1 & 0 \\
0 & 0 & 0 & 0 & 0 & 0 & 0 & 1
\end{array}\right] \times } \\
& {\left[\begin{array}{llllllll}
1 & 1 & 0 & 0 & 0 & 0 & 0 & 0 \\
1 & 0 & 0 & 0 & 0 & 0 & 0 & 0 \\
0 & 0 & 1 & 1 & 0 & 0 & 0 & 0 \\
0 & 0 & 1 & 0 & 0 & 0 & 0 & 0 \\
0 & 0 & 0 & 0 & 1 & 0 & 0 & 0 \\
0 & 0 & 0 & 0 & 0 & 1 & 0 & 0 \\
0 & 0 & 0 & 0 & 0 & 0 & 1 & 0 \\
0 & 0 & 0 & 0 & 0 & 0 & 0 & 1
\end{array}\right], }
\end{aligned}
$$

$$
\text { and } A H_{2}^{-1}(3)=\frac{1}{2}\left[\begin{array}{cccccccc}
0 & 1 & 0 & 0 & 0 & 0 & 0 & 0 \\
1 & -1 & 0 & 0 & 0 & 0 & 0 & 0 \\
0 & 0 & 0 & 1 & 0 & 0 & 0 & 0 \\
0 & 0 & 1 & -1 & 0 & 0 & 0 & 0 \\
0 & 0 & 0 & 0 & 1 & 0 & 0 & 0 \\
0 & 0 & 0 & 0 & 0 & 1 & 0 & 0 \\
0 & 0 & 0 & 0 & 0 & 0 & 1 & 0 \\
0 & 0 & 0 & 0 & 0 & 0 & 0 & 1
\end{array}\right] \times
$$




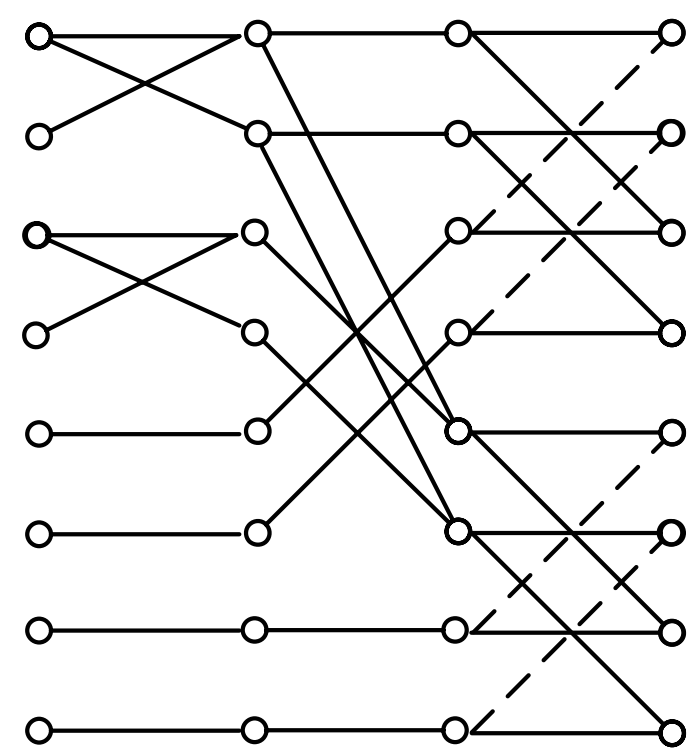

Fig. 1. Fast butterfly diagrams for forward arithmetic-Haar transforms, $\vec{\omega}_{3}=$ $\{0,1,1\}, r=2$ and $n=3$.

$$
\begin{gathered}
{\left[\begin{array}{rrrrrrrr}
1 & 0 & 0 & 0 & 0 & 0 & 0 & 0 \\
0 & 1 & 0 & 0 & 0 & 0 & 0 & 0 \\
-1 & 0 & 0 & 0 & 1 & 0 & 0 & 0 \\
0 & -1 & 0 & 0 & 0 & 1 & 0 & 0 \\
0 & 0 & 1 & 0 & 0 & 0 & 0 & 0 \\
0 & 0 & 0 & 1 & 0 & 0 & 0 & 0 \\
0 & 0 & 0 & 0 & 0 & 0 & 1 & 0 \\
0 & 0 & 0 & 0 & 0 & 0 & 0 & 1
\end{array}\right]} \\
\times\left[\begin{array}{cccccccc}
1 & 0 & 1 & 0 & 0 & 0 & 0 & 0 \\
0 & 1 & 0 & 1 & 0 & 0 & 0 & 0 \\
-1 & 0 & 1 & 0 & 0 & 0 & 0 & 0 \\
0 & -1 & 0 & 1 & 0 & 0 & 0 & 0 \\
0 & 0 & 0 & 0 & 1 & 0 & 1 & 0 \\
0 & 0 & 0 & 0 & 0 & 1 & 0 & 1 \\
0 & 0 & 0 & 0 & -1 & 0 & 1 & 0 \\
0 & 0 & 0 & 0 & 0 & -1 & 0 & 1
\end{array}\right] .
\end{gathered}
$$

The inverse multi-polarity arithmetic-Haar transform $A H_{2}^{-1}(3)$ can be also factorized in a similar way using the fast algorithms. From the representations of $A H_{2}(3)$ and $A H_{2}^{-1}(3)$ by products of the factored matrices, the corresponding fast flow diagrams for the calculation of forward and inverse multi-polarity arithmetic-Haar transforms are shown in Fig. 1 and Fig. 2, accordingly. In all the figures in this article, the solid lines and dotted lines represent addition and subtraction, respectively.

\section{CONCLUSION}

The generalizations of multi-polarity arithmetic-Haar transform are presented in this article. By selecting the polarity and $r$ of the multi-polarity arithmetic-Haar transform, we can find the optimal expression for a given logic function. The new transform shows both the computational advantage and better

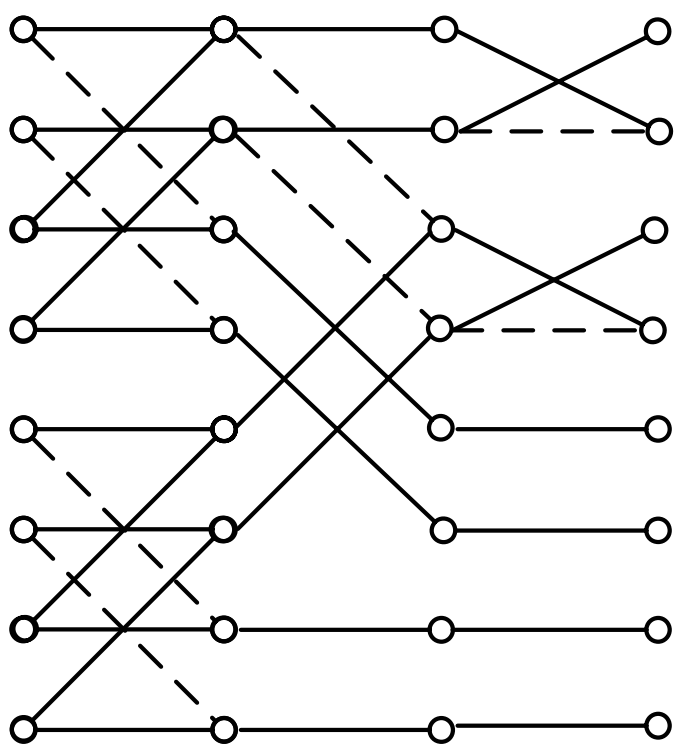

Fig. 2. Fast butterfly diagrams for inverse arithmetic-Haar transforms, $\vec{\omega}_{3}=$ $\{0,1,1\}, r=2$ and $n=3$.

experimental results over Walsh and arithmetic transforms. There are different Decision Diagrams (DDs) that have been used for spectral transforms [2], [7]. Hence, the calculation of this transform through DDs can also be developed, which is more suitable for large discrete functions.

\section{REFERENCES}

[1] B. J. Falkowski, "A note on the polynomial form of Boolean functions and related topics," IEEE Transactions on Computers, vol. 48, no. 8, pp. 860-864, Sept. 1999.

[2] T. Sasao and M. Fujita, Eds., Representation of Discrete Functions. Boston: Kluwer Academic, 1996.

[3] R. S. Stankovic and B. J. Falkowski, "The Haar wavelet transform: its status and achievements," Computers and Electrical Engineering, vol. 29, no. 1, pp. 25-44, Jan. 2003.

[4] B. J. Falkowski, "Mutual relations between Arithmetic and Haar functions," in Proceedings of 31st IEEE International Symposium on Circuits and Systems, vol. 5, Monterey, California, June 1998, pp. 138-141.

[5] R. S. Stankovic, C. Moraga, and J. T. Astola, "From Fourier expansions to Arithmetic-Haar expressions on quaternion groups," Applicable Algebra in Engineering, Communication and Computing, vol. 12, no. 3, pp. 227-253, July 2001

[6] B. J. Falkowski and S. Yan, "Generalization of Arithmetic-Haar transform for higher dimensions," in Proceedings of 47th IEEE International Midwest Symposium on Circuits and Systems, vol. 1, Hiroshima, Japan, July 2004, pp. 513-516.

[7] R. S. Stankovic and J. T. Astola, Spectral Interpretation of Decision Diagram. New York: Springer-Verlag, 2003.

[8] B. J. Falkowski, "Properties and ways of calculation of multi-polarity generalized Walsh transforms," IEEE Transactions on Circuits and Systems-Part II: Analog and Digital Signal Processing, vol. 41, no. 6, pp. 380-391, June 1994.

[9] B. J. Falkowski and M. A. Perkowski, "A family of all essential radix-2 addition/subtraction multi-polarity transforms: algorithms and interpretations in Boolean domain," in Proceedings of 23rd IEEE International Symposium on Circuits and Systems, New Orleans, Louisana, May 1990, pp. 2913-2916.

[10] B. J. Falkowski, "Generalized multi-polarity Haar transform," in Proceedings of 35th IEEE International Symposium on Circuits and Systems, vol. 5, Phoenix, Arizona, May 2002, pp. 757-760. 